\title{
Risk stratification of patients admitted to hospital with covid-19 using the ISARIC WHO Clinical Characterisation Protocol: development and validation of the 4C Mortality Score
}

\author{
Stephen R Knight, ${ }^{1}$ Antonia Ho, ${ }^{2,3}$ Riinu Pius, ${ }^{1}$ Iain Buchan, ${ }^{4}$ Gail Carson, ${ }^{5}$ Thomas M Drake, ${ }^{1}$ \\ Jake Dunning, ${ }^{6,7}$ Cameron J Fairfield, ${ }^{1}$ Carrol Gamble, ${ }^{8}$ Christopher A Green, ${ }^{9}$ Rishi Gupta, ${ }^{10}$ \\ Sophie Halpin, ${ }^{8}$ Hayley E Hardwick, ${ }^{11}$ Karl A Holden, ${ }^{11}$ Peter W Horby, ${ }^{5}$ Clare Jackson, ${ }^{8}$ \\ Kenneth A Mclean, ${ }^{1}$ Laura Merson, ${ }^{5}$ Jonathan S Nguyen-Van-Tam, ${ }^{12}$ Lisa Norman, ${ }^{1}$ \\ Mahdad Noursadeghi, ${ }^{13}$ Piero L Olliaro, ${ }^{14}$ Mark G Pritchard, ${ }^{14}$ Clark D Russell, ${ }^{15}$ \\ Catherine A Shaw, ${ }^{1}$ Aziz Sheikh, ${ }^{1}$ Tom Solomon, ${ }^{11,16}$ Cathie Sudlow, ${ }^{17}$ Olivia V Swann, ${ }^{18}$ \\ Lance CW Turtle, ${ }^{11,19}$ Peter JM Openshaw, ${ }^{7}$ J Kenneth Baillie, ${ }^{20,21}$ Malcolm G Semple, ${ }^{11,22}$ \\ Annemarie B Docherty, ${ }^{1,21}$ Ewen M Harrison, ${ }^{1,23}$ on behalf of the ISARIC 4C investigators
}

For numbered affiliations see end of the article.

Correspondence to: M G Semple m.g.semple@liverpool.ac.uk

(or@ProfCalumSemple on Twitter ORCID 0000-0001-9700-0418)

Additional material is published online only. To view please visit the journal online.

Cite this as: $B M / 2020 ; 370: \mathrm{m} 3339$ http://dx.doi.org/10.1136/bmj.m3339

Accepted: 25 August 2020

\section{ABSTRACT}

\section{OBJECTIVE}

To develop and validate a pragmatic risk score to predict mortality in patients admitted to hospital with coronavirus disease 2019 (covid-19).

\section{DESIGN}

Prospective observational cohort study.

SETTING

International Severe Acute Respiratory and emerging Infections Consortium (ISARIC) World Health Organization (WHO) Clinical Characterisation Protocol UK (CCP-UK) study (performed by the ISARIC Coronavirus Clinical Characterisation ConsortiumISARIC-4C) in 260 hospitals across England, Scotland, and Wales. Model training was performed on a cohort of patients recruited between 6 February and 20 May 2020, with validation conducted on a second cohort of patients recruited after model development between 21 May and 29 June 2020.

PARTICIPANTS

Adults (age $\geq 18$ years) admitted to hospital with covid-19 at least four weeks before final data extraction.

\section{WHAT IS ALREADY KNOWN ON THIS TOPIC}

Robust, validated clinical prediction tools are lacking that identify patients with coronavirus disease 2019 (covid-19) who are at the highest risk of mortality Given the uncertainty about how to stratify patients with covid-19, considerable interest exists in risk stratification scores to support frontline clinical decision making

Available risk stratification tools have a high risk of bias, small sample size resulting in uncertainty, poor reporting, and lack formal validation

\section{WHAT THIS STUDY ADDS}

Most existing covid-19 risk stratification tools performed poorly in our cohort; caution is needed when novel tools based on small patient populations are applied to cohorts in hospital with covid-19

The $4 \mathrm{C}$ (Coronavirus Clinical Characterisation Consortium) Mortality Score is an easy-to-use and valid prediction tool for in-hospital mortality, accurately categorising patients as being at low, intermediate, high, or very high risk of death

This pragmatic and clinically applicable score outperformed other risk stratification tools, showed clinical decision making utility, and had similar performance to more complex models
MAIN OUTCOME MEASURE

In-hospital mortality.

\section{RESULTS}

35463 patients were included in the derivation dataset (mortality rate $32.2 \%$ ) and 22361 in the validation dataset (mortality rate $30.1 \%$ ). The final 4C Mortality Score included eight variables readily available at initial hospital assessment: age, sex, number of comorbidities, respiratory rate, peripheral oxygen saturation, level of consciousness, urea level, and $C$ reactive protein (score range $0-21$ points). The 4C Score showed high discrimination for mortality (derivation cohort: area under the receiver operating characteristic curve $0.79,95 \%$ confidence interval 0.78 to 0.79 ; validation cohort: $0.77,0.76$ to 0.77 ) with excellent calibration (validation: calibrationin-the-large $=0$, slope $=1.0)$. Patients with a score of at least $15(n=4158,19 \%)$ had a $62 \%$ mortality (positive predictive value 62\%) compared with $1 \%$ mortality for those with a score of 3 or less $(n=1650$, $7 \%$; negative predictive value $99 \%$ ). Discriminatory performance was higher than 15 pre-existing risk stratification scores (area under the receiver operating characteristic curve range 0.61-0.76), with scores developed in other covid-19 cohorts often performing poorly (range 0.63-0.73).

\section{CONCLUSIONS}

An easy-to-use risk stratification score has been developed and validated based on commonly available parameters at hospital presentation. The $4 \mathrm{C}$ Mortality Score outperformed existing scores, showed utility to directly inform clinical decision making, and can be used to stratify patients admitted to hospital with covid-19 into different management groups. The score should be further validated to determine its applicability in other populations.

\section{STUDY REGISTRATION}

ISRCTN66726260

\section{Introduction}

Disease resulting from infection with severe acute respiratory syndrome coronavirus 2 (SARS-CoV-2) has a high mortality rate with deaths predominantly caused by respiratory failure. ${ }^{1}$ As of 1 September 2020 , 
over 25 million people had confirmed coronavirus disease 2019 (covid-19) worldwide and at least 850000 people had died from the disease. ${ }^{2}{ }^{3}$ As hospitals around the world are faced with an influx of patients with covid-19, there is an urgent need for a pragmatic risk stratification tool that will allow the early identification of patients infected with SARSCoV-2 who are at the highest risk of death to guide management and optimise resource allocation.

Prognostic scores attempt to transform complex clinical pictures into tangible numerical values. Prognostication is more difficult when dealing with a severe pandemic illness such as covid-19 because strain on healthcare resources and rapidly evolving treatments alter the risk of death over time. Early information has suggested that the clinical course of a patient with covid-19 is different from that of pneumonia, seasonal influenza, or sepsis. ${ }^{4}$ Most patients with severe covid-19 have developed a clinical picture characterised by pneumonitis, profound hypoxia, and systemic inflammation affecting multiple organs. $^{1}$

A recent review identified many prognostic scores used for covid-19, ${ }^{5}$ which varied in their setting, predicted outcome measure, and the clinical parameters included. The large number of risk stratification tools reflects difficulties in their application, with most scores showing moderate performance at best and no benefit to clinical decision making. ${ }^{67}$ Many novel covid-19 prognostic scores have been found to have a high risk of bias, which could reflect development in small cohorts, and many have been published without clear details of model derivation and testing. ${ }^{5}$ Therefore, a risk stratification tool within a large national cohort of patients admitted to hospital with covid-19 is needed with clear development and validation details.

Our aim was to develop and validate a pragmatic, clinically relevant risk stratification score that uses routinely available clinical information at hospital presentation to predict in-hospital mortality in patients admitted to hospital with covid-19. We then aimed to compare this score with existing prognostic models.

\section{Methods}

\section{Study design and setting}

The International Severe Acute Respiratory and emerging Infections Consortium (ISARIC) World Health Organization (WHO) Clinical Characterisation Protocol UK (CCP-UK) study is an ongoing prospective cohort study. The study is being performed by the ISARIC Coronavirus Clinical Characterisation Consortium (ISARIC-4C) in 260 hospitals across England, Scotland, and Wales (National Institute for Health Research Clinical Research Network Central Portfolio Management System ID 14152). The protocol and further study details are available online. ${ }^{8}$ Model development and reporting followed the TRIPOD (transparent reporting of a multivariable prediction model for individual prediction or diagnosis) guidelines. ${ }^{9}$ The study is being conducted according to a predefined protocol (appendix 1).

\section{Participants}

The study recruited consecutive patients aged 18 years and older with a completed index admission to one of 260 hospitals in England, Scotland, or Wales. ${ }^{8}$ Reverse transcriptase polymerase chain reaction was the only mode of testing available during the period of study. The decision to test was at the discretion of the clinician attending the patient, and not defined by protocol. The enrolment criterion "high likelihood of infection" reflected that a preparedness protocol cannot assume a diagnostic test will be available for an emergent pathogen. In this activation, site training emphasised the importance of only recruiting proven cases.

\section{Data collection}

Demographic, clinical, and outcome data were collected by using a prespecified case report form. Comorbidities were defined according to a modified Charlson comorbidity index. ${ }^{10}$ Comorbidities collected were chronic cardiac disease, chronic respiratory disease (excluding asthma), chronic renal disease (estimated glomerular filtration rate $\leq 30$ ), mild to severe liver disease, dementia, chronic neurological conditions, connective tissue disease, diabetes mellitus (diet, tablet, or insulin controlled), HIV or AIDS, and malignancy. These conditions were selected a priori by a global consortium to provide rapid, coordinated clinical investigation of patients presenting with any severe or potentially severe acute infection of public interest and enabled standardisation.

Clinician defined obesity was also included as a comorbidity owing to its probable association with adverse outcomes in patients with covid-19. ${ }^{11} 12$ The clinical information used to calculate prognostic scores was taken from the day of admission to hospital. ${ }^{13}$ A practical approach was taken to sample size requirements. ${ }^{14}$ We used all available data to maximise the power and generalisability of our results. Model reliability was assessed by using a temporally distinct validation cohort with geographical subsetting, together with sensitivity analyses.

\section{Outcomes}

The primary outcome was in-hospital mortality. This outcome was selected because of the importance of the early identification of patients likely to develop severe illness from SARS-CoV-2 infection (a rule in test). We chose to restrict analysis of outcomes to patients who were admitted more than four weeks before final data extraction (29 June 2020) to enable most patients to complete their hospital admission.

\section{Independent predictor variables}

A reduced set of potential predictor variables was selected a priori, including patient demographic information, common clinical investigations, and parameters consistently identified as clinically important in covid-19 cohorts following the methods described by Wynants and colleagues (appendix 2). ${ }^{5}$ Candidate predictor variables were selected based on 
three common criteria ${ }^{15}$ : patient and clinical variables known to influence outcome in pneumonia and flulike illness; clinical biomarkers previously identified within the literature as potential predictors in patients with covid-19; values available for at least two thirds of patients within the derivation cohort.

Because our overall aim was to develop an easy-touse risk stratification score, we made the decision to include an overall comorbidity count for each patient within model development giving each comorbidity equal weight, rather than individual comorbidities. Recent evidence suggests an additive effect of comorbidity in patients with covid-19, with increasing number of comorbidities associated with poorer outcomes. $^{16}$

\section{Model development}

Missing values for potential candidate variables were handled by using multiple imputation with chained equations, under the missing at random assumption (appendix 6). Ten sets, each with 10 iterations, were imputed using available explanatory variables for both cohorts (derivation and validation). The outcome variable was included as a predictor in the derivation dataset but not the validation dataset. All model derivation and validation was performed in imputed datasets, with Rubin's rules ${ }^{17}$ used to combine results.

Models were trained by using all available data up to 20 May 2020. The primary intention was to create a pragmatic model for bedside use not requiring complex equations, online calculators, or mobile applications. An a priori decision was therefore made to categorise continuous variables in the final prognostic score.

We used a three stage model building process (fig 1). Firstly, generalised additive models were built incorporating continuous smoothed predictors (penalised thin plate splines) in combination with categorical predictors as linear components. A criterion based approach to variable selection was taken based on the deviance explained, the unbiased risk estimator, and the area under the receiver operating characteristic curve. Secondly, we visually inspected plots of component smoothed continuous predictors for linearity, and selected optimal cut-off values by using the methods of Barrio and colleagues. ${ }^{18}$

Lastly, final models using categorised variables were specified with least absolute shrinkage and selection operator logistic regression. L1 penalised coefficients were derived using 10 -fold cross validation to select the value of lambda (minimised cross validated sum of squared residuals). We converted shrunk coefficients to a prognostic index with appropriate scaling to create the pragmatic 4C Mortality Score (where 4C stands for Coronavirus Clinical Characterisation Consortium).

We used machine learning approaches in parallel for comparison of predictive performance. Given issues with interpretability, this was intended to provide a best-in-class comparison of predictive performance when accounting for any complex underlying interactions. Gradient boosting decision trees were used (XGBoost). All candidate predictor variables identified were included within the model, except for those with high missing values $(>33 \%)$. We retained individual major comorbidity variables within the model to determine whether inclusion improved predictive performance. An 80\%/20\% random split of the derivation dataset was used to define train and test sets. The validation datasets were held back and not used in the training process. We used a mortality label and design matrix of centred or standardised continuous and categorical variables including all candidate variables to train gradient boosted trees minimising the binary classification error rate (defined as number of wrong cases divided by number of all cases). Hyperparameters were tuned, including the learning rate and maximum tree depth, to maximise the area under the receiver operating characteristic curve in the test set. This approach affords flexibility in the handling of missing data; therefore, two models were trained and optimised, one using imputed data and the other modelling missingness in complete case data.

We assessed discrimination for all models by using the area under the receiver operating characteristic curve in the derivation cohort, with $95 \%$ confidence intervals calculated by bootstrapped resampling (2000 samples). A value of 0.5 indicates no predictive ability, 0.8 is considered good, and 1.0 is perfect. ${ }^{19}$ We assessed overall goodness of fit with the Brier score, ${ }^{20}$ a measure to quantify how close predictions are to the truth. The score ranges between 0 and 1 , where smaller values indicate superior model performance. We plotted model calibration curves to examine agreement between predicted and observed risk across deciles of mortality risk to determine the presence of over or under prediction. Risk cut-off values were defined by the total point score for an individual, which represented low ( $<2 \%$ mortality rate), intermediate (2-14.9\%), or high risk $(\geq 15 \%)$ groups, similar to commonly used pneumonia risk stratification scores. ${ }^{21} 22$

We performed sensitivity analyses by using complete case data. Model discrimination was also checked in ethnic groups and by sex using imputed datasets.

\section{Model validation}

Patients entered into the ISARIC WHO CCP-UK study after 20 May 2020 were included in a separate validation cohort (fig 1). We determined discrimination, calibration, and performance across a range of clinically relevant metrics. To avoid bias in the assessment of outcomes, patients who were admitted within four weeks of data extraction on 29 June 2020 were excluded. We included patients without an outcome after four weeks and considered to have had no event.

A sensitivity analysis was also performed, with stratification of the validation cohort by geographical location. We selected this geographical categorisation based on well described economic and health inequalities between the north and south of the United Kingdom. ${ }^{2324}$ Recent analysis has shown the impact of deprivation on risk of dying with covid-19. ${ }^{25}$ As a result, 


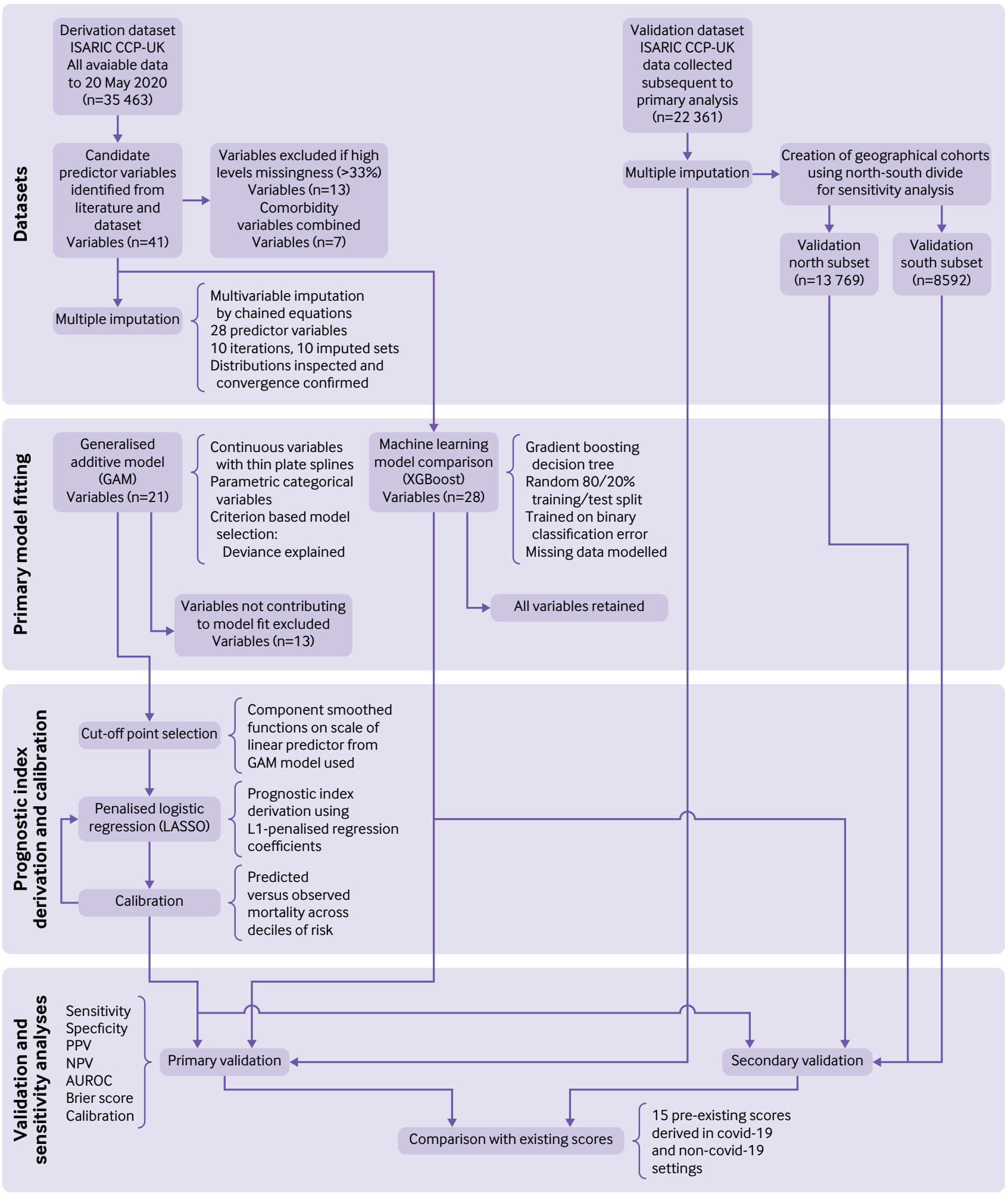

Fig 1 | Model derivation and validation workflow. AUROC=area under the receiver operating characteristic curve; covid-19=coronavirus disease 2019; ISARIC CCP-UK=International Severe Acute Respiratory and emerging Infections Consortium Clinical Characterisation Protocol UK; $\mathrm{NPV}=$ negative predictive value; $\mathrm{PPV}=$ positive predictive value

population differences between regions could change the discriminatory performance of risk stratification scores. Two geographical cohorts were created, based on north-south geographical locations across the UK as defined by Hacking and colleagues. ${ }^{23}$ We performed a further sensitivity analysis to determine model performance in ethnic minority groups given the reported differences in covid-19 outcomes. ${ }^{26}$ 
All tests were two tailed and $\mathrm{P}$ values less than 0.05 were considered statistically significant. We used $\mathrm{R}$ (version 3.6.3) with the finalfit, mice, glmnet, pROC, recipes, xgboost, rmda, and tidyverse packages for all statistical analysis.

\section{Comparison with existing risk stratification scores}

All derived models in the derivation dataset were compared within the validation cohort with existing scores. We assessed model performance by using the area under the receiver operating characteristic curve statistic, sensitivity, specificity, positive predictive value, and negative predictive value. Existing risk stratification scores were identified through a systematic literature search of Embase, WHO Medicus, and Google Scholar databases. We used the search terms "pneumonia," "sepsis," "influenza," "COVID-19," "SARS-CoV-2," "coronavirus” combined with "score” and "prognosis." We applied no language or date restrictions. The last search was performed on 1 July 2020. Risk stratification tools were included whose variables were available within the database and had accessible methods for calculation.

We calculated performance characteristics according to original publications, and selected score cutoff values for adverse outcomes based on the most commonly used criteria identified within the literature. Cut-off values were the score value for which the patient was considered at low or high risk of adverse outcome, as defined by the study authors. Patients with one or more missing input variables were omitted for that particular score.

We also performed a decision curve analysis. ${ }^{27}$ Briefly, assessment of the adequacy of clinical prediction models can be extended by determining clinical utility. By using decision curve analysis, we can make a clinical judgment about the relative value of benefits (treating a true positive) and harms (treating a false positive) associated with a clinical prediction tool. The standardised net benefit was plotted against the threshold probability for considering a patient high risk for age alone and for the best discriminating models applicable to more than $50 \%$ of patients in the validation cohort.

\section{Patient and public involvement}

This was an urgent public health research study in response to a Public Health Emergency of International Concern. Patients or the public were not involved in the design, conduct, or reporting of this rapid response research.

\section{Results}

We collected data from 35463 patients between 6 February 2020 and 20 May 2020 in the derivation cohort; 1275 (3.6\%) patients had no outcome recorded and were considered alive. The overall mortality rate was $32.2 \%$ (11426 patients). The median age of patients in the cohort was 73 years (interquartile range $59-83$ ); $41.7 \%$ (14741) were female and 76.0\% (26966) had at least one comorbidity. Table 1 shows demographic and clinical characteristics for the derivation and validation datasets.

\section{Model development}

We identified 41 candidate predictor variables measured at hospital admission for model creation (fig 1, appendix 2). After the creation of a composite variable containing all seven individual comorbidities and the exclusion of 13 variables owing to high levels of missing values, 21 variables remained.

We identified eight important predictors of mortality by using generalised additive modelling with multiply imputed datasets: age, sex, number of comorbidities, respiratory rate, peripheral oxygen saturation, Glasgow coma scale, urea level, and $\mathrm{C}$ reactive protein (for variable selection process, see appendix 3). Given the need for a pragmatic score for use at the bedside, continuous variables were converted to factors with cut-off values chosen by using component smoothed functions (on linear predictor scale) from generalised additive modelling (appendix 4).

On entering variables into a penalised logistic regression model (least absolute shrinkage and selection operator), all variables were retained within the final model (appendix 5). We converted penalised regression coefficients into a prognostic index by using appropriate scaling (4C Mortality Score range 0-21 points; table 2).

The 4C Mortality Score showed good discrimination for death in hospital within the derivation cohort (table 3), with performance approaching that of the XGBoost model. The 4C Mortality Score showed good calibration (calibration intercept $=0$, slope $=1$, Brier score 0.170 ) across the range of risk and no adjustment to the model was required (appendix 11).

\section{Model validation}

The validation cohort included data from 22361 patients collected between 21 May 2020 and 29 June 2020 who had at least four weeks of follow-up; 743 (3.3\%) patients had no outcome recorded and were considered alive. The overall mortality rate was $30.1 \%$ (6729 patients). The median age of patients in the cohort was 76 (interquartile range 60-85) years; 10178 (45.6\%) were female and 17263 (77\%) had at least one comorbidity (table 1).

Discrimination of the $4 \mathrm{C}$ Mortality Score in the validation cohort was similar to that of the XGBoost model (table 3). Calibration was also found to be excellent in the validation cohort: overall observed $(30.1 \%)$ versus predicted $(30.1 \%)$ mortality was equal (calibration-in-the-large $=0$ ) and calibration was excellent over the range of risk (slope $=1$, Brier score 0.171; fig 2). The 4C Mortality Score showed good performance in clinically relevant metrics across a range of cut-off values (table 4).

Four risk groups were defined with corresponding mortality rates determined (table 5 ): low risk (0-3 score, mortality rate $1.2 \%$ ), intermediate risk (4-8 score, 9.9\%), high risk (9-14 score, 31.4\%), and very high risk ( $\geq 15$ score, $61.5 \%$ ). Performance metrics showed a 


\begin{tabular}{|c|c|c|c|c|}
\hline \multirow[b]{2}{*}{ Characteristics } & \multicolumn{2}{|c|}{ Derivation cohort } & \multicolumn{2}{|c|}{ Validation cohort } \\
\hline & $\begin{array}{l}\text { No of patients (\%) } \\
\text { or median (IQR) }\end{array}$ & Total No (\%) & $\begin{array}{l}\text { No of patients }(\%) \\
\text { or median (IQR) }\end{array}$ & Total No (\%) \\
\hline Mortality in hospital & $11426(32.2)$ & $35463(100.0)$ & $6729(30.1)$ & $22361(100.0)$ \\
\hline \multicolumn{5}{|l|}{ Age (years) } \\
\hline$<50$ & $4876(13.8)$ & $35277(99.5)$ & $2808(12.6)$ & $22361(100.0)$ \\
\hline $50-69$ & $10183(28.9)$ & - & $5762(25.8)$ & - \\
\hline $70-79$ & $8017(22.7)$ & - & $4951(22.1)$ & - \\
\hline$\geq 80$ & $12201(34.6)$ & - & $8840(39.5)$ & - \\
\hline \multicolumn{5}{|l|}{ Sex at birth } \\
\hline Female & $14741(41.7)$ & $35356(99.7)$ & $10178(45.6)$ & $22319(99.8)$ \\
\hline \multicolumn{5}{|l|}{ Ethnicity } \\
\hline White & $26300(82.2)$ & $31987(90.2)$ & $16831(84.9)$ & $19818(88.6)$ \\
\hline South Asian & $1647(5.1)$ & - & $811(4.1)$ & - \\
\hline East Asian & $271(0.8)$ & - & $140(0.7)$ & - \\
\hline Black & $1256(3.9)$ & - & 769 (3.9) & - \\
\hline Other ethnic minority & $2513(7.9)$ & - & $1267(6.4)$ & - \\
\hline Chronic cardiac disease & $10513(31.8)$ & $33090(93.3)$ & 7019 (34.0) & $20616(92.2)$ \\
\hline Chronic kidney disease & $5653(17.2)$ & $32834(92.6)$ & 3769 (18.4) & $20444(91.4)$ \\
\hline Malignant neoplasm & $3312(10.2)$ & $32556(91.8)$ & $2187(10.8)$ & $20297(90.8)$ \\
\hline Moderate or severe liver disease & 604 (1.9) & $32538(91.8)$ & $434(2.1)$ & $20218(90.4)$ \\
\hline Obesity (clinician defined) & 3414 (11.4) & $29829(84.1)$ & $2234(12.2)$ & $18304(81.9)$ \\
\hline Chronic pulmonary disease (not asthma) & $5830(17.7)$ & $32990(93.0)$ & 3737 (18.2) & $20502(91.7)$ \\
\hline Diabetes (type 1 and 2) & $8487(26.0)$ & $32622(92.0)$ & $4275(21.9)$ & $19511(87.3)$ \\
\hline \multicolumn{5}{|l|}{ No of comorbidities } \\
\hline 0 & $8497(24.0)$ & $35463(100.0)$ & $5098(22.8)$ & $22361(100.0)$ \\
\hline 1 & $9941(28.0)$ & - & $6114(27.3)$ & - \\
\hline$\geq 2$ & $17025(48.0)$ & - & 11149 (49.9) & - \\
\hline Respiratory rate (breaths/min) & $22.0(9.0)$ & $33330(94.0)$ & $20.0(8.0)$ & $20970(93.8)$ \\
\hline Oxygen saturation (\%) & $94.0(6.0)$ & $33696(95.0)$ & $94.0(5.0)$ & $21197(94.8)$ \\
\hline Systolic blood pressure $(\mathrm{mm} \mathrm{Hg})$ & $124.0(33.0)$ & 33637 (94.9) & $129.0(33.0)$ & $21073(94.2)$ \\
\hline Diastolic blood pressure $(\mathrm{mm} \mathrm{Hg})$ & $70.0(19.0)$ & $33568(94.7)$ & $73.0(20.0)$ & $21026(94.0)$ \\
\hline Temperature $\left({ }^{\circ} \mathrm{C}\right)$ & $37.3(1.5)$ & $33467(94.4)$ & $37.1(1.5)$ & $21139(94.5)$ \\
\hline Heart rate (bpm) & $90.0(27.0)$ & $33405(94.2)$ & $90.0(28.0)$ & 20991 (93.9) \\
\hline Glasgow coma scale score & $15.0(0.0)$ & $30819(86.9)$ & $15.0(0.0)$ & $20015(89.5)$ \\
\hline Haemoglobin $(\mathrm{g} / \mathrm{L})$ & $129.0(30.0)$ & $29924(84.4)$ & $127.0(31.0)$ & $18480(82.6)$ \\
\hline White blood cell count $\left(10^{9} / \mathrm{L}\right)$ & $7.4(5.1)$ & $29740(83.9)$ & $7.6(5.3)$ & $18362(82.1)$ \\
\hline Neutrophil count $\left(10^{9} / \mathrm{L}\right)$ & $5.6(4.6)$ & 29594 (83.5) & $5.8(4.9)$ & $18354(82.1)$ \\
\hline Lymphocyte count $\left(10^{9} / \mathrm{L}\right)$ & $0.9(0.7)$ & $29553(83.3)$ & $0.9(0.7)$ & $18348(82.1)$ \\
\hline Platelet count $\left(10^{9} / \mathrm{L}\right)$ & $216.0(120.0)$ & $29582(83.4)$ & $223.0(126.0)$ & $18281(81.8)$ \\
\hline Sodium (mmol/L) & $137.0(6.0)$ & $29522(83.2)$ & $137.0(6.0)$ & $18409(82.3)$ \\
\hline Potassium (mmol/L) & $4.1(0.8)$ & $27224(76.8)$ & $4.1(0.8)$ & $16926(75.7)$ \\
\hline Total bilirubin (mg/dL) & $10.0(7.0)$ & $24446(68.9)$ & $10.0(7.0)$ & $15404(68.9)$ \\
\hline Urea (mmol/L) & $7.0(6.3)$ & $26122(73.7)$ & $7.3(6.8)$ & $16863(75.4)$ \\
\hline Creatinine $(\mu \mathrm{mol} / \mathrm{L})$ & $86.0(53.0)$ & $29439(83.0)$ & $86.0(56.0)$ & $18225(81.5)$ \\
\hline C reactive protein $(\mathrm{mg} / \mathrm{L})$ & $84.9(122.0)$ & $27856(78.5)$ & $78.0(120.0)$ & $17119(76.6)$ \\
\hline
\end{tabular}

Covid-19=coronavirus disease 2019; IQR=interquartile range.

Comorbidities were defined using the Charlson comorbidity index, with the addition of clinician defined obesity.

high sensitivity (99.7\%) and negative predictive value (98.8\%) for the low risk group, covering $7.4 \%$ of the cohort and a corresponding mortality rate of $1.2 \%$.

Patients in the intermediate risk group (score 4-8, $\mathrm{n}=4889,21.9 \%$ ) had a mortality rate of $9.9 \%$ (negative predictive value $90.1 \%$ ). Patients in the high risk group (score 9-14, $\mathrm{n}=11664,52.2 \%$ ) had a mortality rate of $31.4 \%$ (negative predictive value $68.6 \%$ ), while patients scoring 15 or higher $(\mathrm{n}=4158,18.6 \%)$ had a mortality rate of $61.5 \%$ (positive predictive value $61.5 \%$ ). An interactive infographic is available at https://isaric4c.net/risk

\section{Comparison with existing tools}

We performed a systematic literature search and identified 15 risk stratification scores that could be applied to these data. ${ }^{622} 28-40$ The $4 \mathrm{C}$ Mortality Score compared well against these existing risk stratification scores in predicting in-hospital mortality (table 6, fig 3 , upper panel). Risk stratification scores originally validated in patients with community acquired pneumonia $(n=9)$ generally had higher discrimination for inhospital mortality in the validation cohort (eg, A-DROP (area under the receiver operating characteristic curve $0.74,95 \%$ confidence interval 0.73 to 0.74 ) and E-CURB65 (0.76, 0.74 to 0.79)) than those developed within covid-19 cohorts $(n=4$ : Surgisphere $(0.63,0.62$ to 0.64 ), DL score (0.67, 0.66 to 0.68 ), COVID-GRAM $(0.71,0.68$ to 0.74$)$, and Xie score $(0.73,0.70$ to 0.75)). Performance metrics for the $4 \mathrm{C}$ Mortality Score compared well against existing risk stratification scores at specified cut-off values (appendix 13). 


\begin{tabular}{|c|c|}
\hline Variable & 4C Mortality Score \\
\hline \multicolumn{2}{|c|}{ Age (years) } \\
\hline$<50$ & - \\
\hline $50-59$ & +2 \\
\hline $60-69$ & +4 \\
\hline $70-79$ & +6 \\
\hline$\geq 80$ & +7 \\
\hline \multicolumn{2}{|c|}{ Sex at birth } \\
\hline Female & - \\
\hline Male & +1 \\
\hline \multicolumn{2}{|c|}{ No of comorbidities* } \\
\hline 0 & - \\
\hline 1 & +1 \\
\hline$\geq 2$ & +2 \\
\hline \multicolumn{2}{|c|}{ Respiratory rate (breaths/min) } \\
\hline$<20$ & - \\
\hline $20-29$ & +1 \\
\hline$\geq 30$ & +2 \\
\hline \multicolumn{2}{|c|}{ Peripheral oxygen saturation on room air (\%) } \\
\hline$\geq 92$ & - \\
\hline$<92$ & +2 \\
\hline \multicolumn{2}{|c|}{ Glasgow coma scale score } \\
\hline 15 & - \\
\hline$<15$ & +2 \\
\hline \multicolumn{2}{|c|}{ Urea $(\mathrm{mmol} / \mathrm{L})$} \\
\hline$<7$ & - \\
\hline $7-14$ & +1 \\
\hline$>14$ & +3 \\
\hline \multicolumn{2}{|c|}{ C reactive protein (mg/L) } \\
\hline$<50$ & - \\
\hline $50-99$ & +1 \\
\hline$\geq 100$ & +2 \\
\hline
\end{tabular}

Covid-19=coronavirus disease 2019

*Comorbidities were defined by using Charlson comorbidity index, with the addition of clinician defined obesity.

The number of patients in whom risk stratification scores could be applied differed owing to certain variables not being available, either because of missingness or because they were not tested for or recorded in clinical practice. Seven scores could be applied to fewer than 2000 patients $(<10 \%)$ in the validation cohort owing to the requirement for biomarkers or physiological parameters that were not routinely captured (eg, lactate dehydrogenase). Decision curve analysis showed that the 4C Mortality Score had better clinical utility across a wide range of threshold risks compared with the best performing existing scores applicable to more than $50 \%$ of the validation cohort (A-DROP and CURB65; fig 3, lower panel).

\section{Sensitivity analysis}

Sensitivity analyses that used complete case data showed similar discrimination (appendix 7) and performance metrics (appendices 8 and 9) to analyses that used the imputed dataset. After stratification of the validation cohort into two geographical cohorts (validation north and south; appendix 14), discrimination remained similar for the 4C Mortality Score in the north subset (area under the receiver operating characteristic curve $0.77,95 \%$ confidence interval 0.76 to 0.78$)$ and south subset $(0.76,0.75$ to 0.77; appendix 6).

Finally, we checked discrimination of the $4 \mathrm{C}$ Mortality Score by sex and ethnic group (appendix 10). Discrimination was the same in men (area under the receiver operating characteristic curve $0.77,95 \%$ confidence interval 0.76 to 0.78$)$ and women (0.76, 0.75 to 0.77 ). Discrimination was better in all nonwhite ethnic groups compared with the white group: South Asian (0.82, 0.80 to 0.85), East Asian (0.85, 0.79 to 0.91$)$, Black $(0.83,0.80$ to 0.86$)$, and other ethnic minority $(0.81,0.79$ to 0.84$)$.

\section{Discussion}

\section{Principal findings}

We have developed and validated the eight variable 4C Mortality Score in a UK prospective cohort study of 57824 patients admitted to hospital with covid-19. The 4C Mortality Score uses patient demographics, clinical observations, and blood parameters that are commonly available at the time of hospital admission and can accurately characterise the population of patients at high risk of death in hospital. The score compared favourably with other models, including best-in-class machine learning techniques, and showed consistent performance across the validation cohorts, including good clinical utility in a decision curve analysis.

Model performance compared well against other generated models, with minimal loss in discrimination despite its pragmatic nature. A machine learning approach showed a marginal improvement in discrimination, but at the cost of interpretability, the requirement for many more input variables, and the need for an app or website calculator that might limit use at the bedside given personal protective equipment requirements. The $4 \mathrm{C}$ Mortality Score showed good applicability within the validation cohort and consistency across all performance measures.

\section{Comparison with other studies}

The 4C Mortality Score contains parameters reflecting patient demographics, comorbidity, physiology, and inflammation at hospital admission; it shares characteristics with existing prognostic scores for

\begin{tabular}{|c|c|c|c|c|}
\hline \multirow[b]{2}{*}{ Model } & \multicolumn{2}{|c|}{ Derivation cohort } & \multicolumn{2}{|c|}{ Validation cohort } \\
\hline & AUROC $(95 \% \mathrm{Cl})$ & Brier score & AUROC $(95 \% \mathrm{Cl})$ & Brier score \\
\hline 4C Mortality Score & 0.786 (0.781 to 0.790$)$ & 0.170 & 0.767 (0.760 to 0.773$)$ & 0.171 \\
\hline Machine learning comparison* & 0.796 (0.786 to 0.807$)$ & 0.191 & $0.779(0.772$ to 0.785$)$ & 0.197 \\
\hline
\end{tabular}




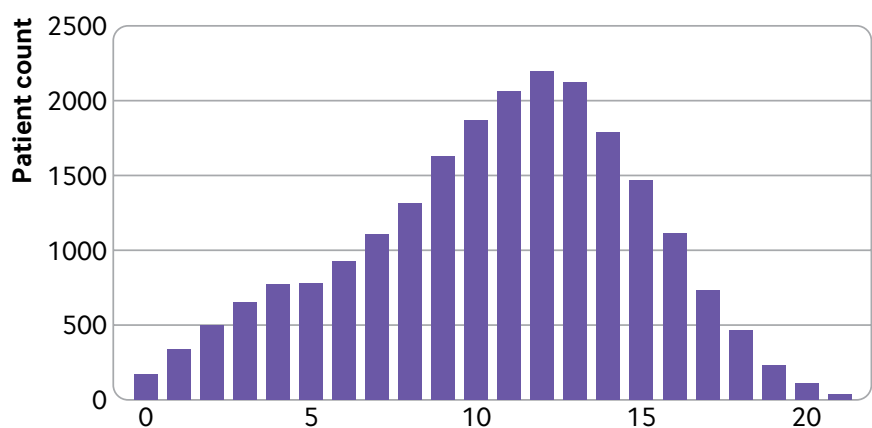

Score value
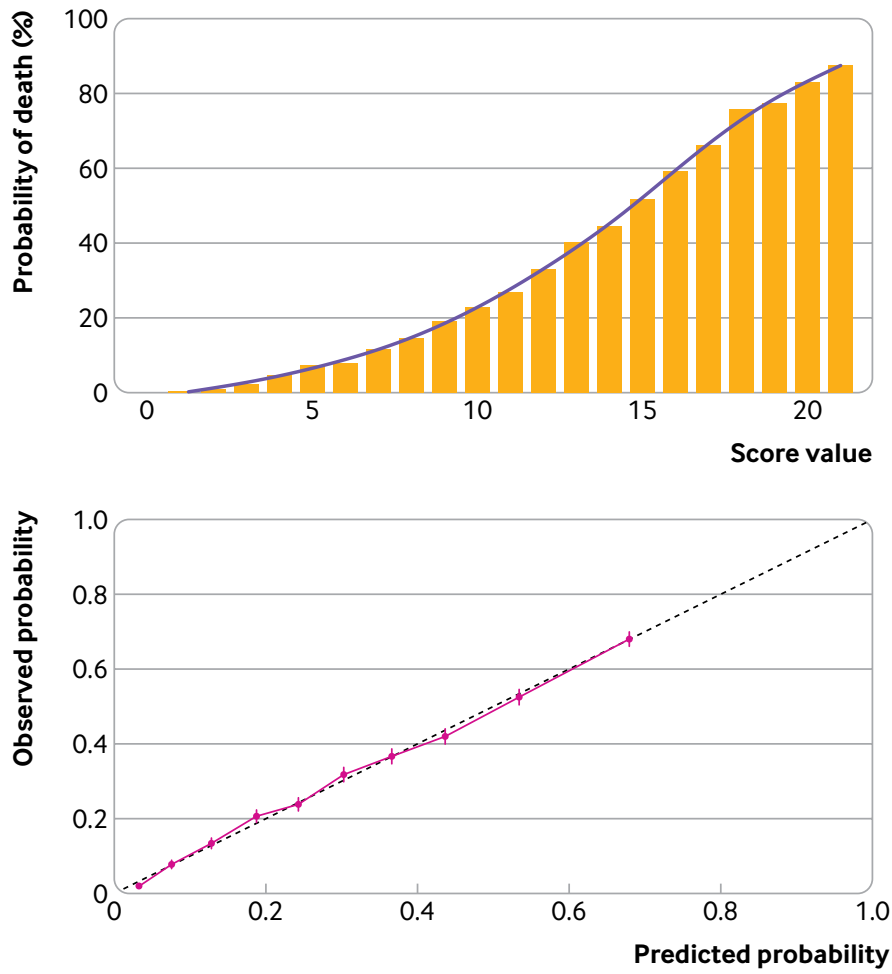

Fig 2 | Upper panel: distribution of patients across range of 4C Mortality Score in validation cohort; middle panel: observed in-hospital mortality across range of $4 \mathrm{C}$ Mortality Score in validation cohort; lower panel: predicted versus observed probability of in-hospital mortality (calibration; red line) for $4 \mathrm{C}$ Mortality Score within validation cohort sepsis and community acquired pneumonia but has important differences as well. No preexisting score appears to use this combination of variables and weightings. Altered consciousness and high respiratory rate are included in most risk stratification scores for sepsis and community acquired pneumonia, $\begin{array}{lllllll}21 & 22 & 28 & 29 & 32 & 33 & 36\end{array}$ while raised urea is also a common component. ${ }^{21} 2228$ Increasing age is a strong predictor of in-hospital mortality in our cohort of patients admitted with covid-19 and is commonly included in other existing covid-19 scores, ${ }^{374142}$ together with comorbidity ${ }^{374142}$ and raised $C$ reactive protein. ${ }^{40} 43$

Discriminatory performance of existing covid-19 scores applied to our cohort was lower than reported in derivation cohorts (DL score 0.74, COVID-GRAM 0.88, Xie score 0.98). ${ }^{373840}$ The use of small inpatient cohorts from Wuhan, China for model development might have resulted in overfitting, limiting generalisability in other cohorts. ${ }^{3840}$ The Xie score demonstrated the highest discriminatory power (0.73), and included age, lymphocyte count, lactate dehydrogenase, and peripheral oxygen saturations. However, we were only able to apply this score for less than $10 \%$ of the validation cohort because lactate dehydrogenase is not routinely measured on hospital admission in the UK.

Owing to challenges of clinical data collection during an epidemic, missing data are common, with choice of predictors influenced by data availability. ${ }^{40}$ Complete case analysis often leads to exclusion of a substantial proportion of the original sample, subsequently leading to a loss of precision and power. ${ }^{44}$ However, the assessment of missing data on model performance in novel covid-19 risk stratification scores has been limited $^{37}$ or unexplored, ${ }^{38}{ }^{40}$ potentially introducing bias and further limiting generalisability to other cohorts. We found discriminatory performance in both derivation and validation cohorts remained similar after the imputation of a wide range of variables, ${ }^{41}$ further supporting the validity of our findings.

The presence of comorbidities is handled differently in covid-19 prognostic scores; comorbidities might be included individually, ${ }^{40}{ }^{42}$ given equal weight, ${ }^{37}$ or found to have no predictive effect. ${ }^{38}$ Recent evidence suggests an additive effect of comorbidity

\begin{tabular}{|c|c|c|c|c|c|c|c|c|c|c|}
\hline Cut-off value & No of patients (\%) & TP & TN & FP & FN & Sensitivity (\%) & Specificity (\%) & PPV (\%) & NPV (\%) & Mortality (\%) \\
\hline \multicolumn{11}{|c|}{ Rule out mortality } \\
\hline$\leq 2$ & $1001(4.5)$ & 6724 & 996 & 14636 & 5 & 99.9 & 6.4 & 31.5 & 99.5 & 0.5 \\
\hline$\leq 3$ & $1650(7.4)$ & 6709 & 1630 & 14002 & 20 & 99.7 & 10.4 & 32.4 & 98.8 & 1.2 \\
\hline$\leq 4$ & $2420(10.8)$ & 6672 & 2363 & 13269 & 57 & 99.2 & 15.1 & 33.5 & 97.6 & 2.4 \\
\hline$\leq 6$ & $4121(18.4)$ & 6542 & 3934 & 11698 & 187 & 97.2 & 25.2 & 35.9 & 95.5 & 4.5 \\
\hline$\leq 8$ & $6539(29.2)$ & 6223 & 6033 & 9599 & 506 & 92.5 & 38.6 & 39.3 & 92.3 & 7.7 \\
\hline$\leq 9$ & $8167(36.5)$ & 5911 & 7349 & 8283 & 818 & 87.8 & 47 & 41.6 & 90.0 & 10.0 \\
\hline \multicolumn{11}{|c|}{ Rule in mortality } \\
\hline$\geq 9$ & $15822(70.8)$ & 6223 & 6033 & 9599 & 506 & 92.5 & 38.6 & 39.3 & 92.3 & 39.3 \\
\hline$\geq 11$ & $12325(55.1)$ & 5483 & 8790 & 6842 & 1246 & 81.5 & 56.2 & 44.5 & 87.6 & 44.5 \\
\hline$\geq 13$ & 8069 (36.1) & 4206 & 11769 & 3863 & 2523 & 62.5 & 75.3 & 52.1 & 82.3 & 52.1 \\
\hline$\geq 15$ & $4158(18.6)$ & 2557 & 14031 & 1601 & 4172 & 38 & 89.8 & 61.5 & 77.1 & 61.5 \\
\hline$\geq 17$ & $1579(7.1)$ & 1142 & 15195 & 437 & 5587 & 17 & 97.2 & 72.3 & 73.1 & 72.3 \\
\hline$\geq 19$ & $381(1.7)$ & 305 & 15556 & 76 & 6424 & 4.5 & 99.5 & 80.1 & 70.8 & 80.1 \\
\hline
\end{tabular}

$\mathrm{FN}=$ false negative; $\mathrm{FP}=$ false positive; $\mathrm{NPV}=$ negative predictive value; $\mathrm{PPV}=$ positive predictive value; $\mathrm{TN}=$ true negative; $\mathrm{TP}=$ true positive. 


\begin{tabular}{llllll}
$\begin{array}{l}\text { Table } 5 \text { | Comparison of mortality rates for } \\
\text { derivation and validation cohorts }\end{array}$ & \multicolumn{2}{c}{ Derivation cohort } & & \multicolumn{2}{c}{ Validation cohort } \\
\cline { 2 - 3 } \cline { 5 - 6 } Risk group & No of patients (\%) & No of deaths (\%) & & No of patients (\%) & No of deaths (\%) \\
Low (0-3) & $2574(7.3)$ & $45(1.7)$ & & $1650(7.4)$ & $20(1.2)$ \\
\hline Intermediate (4-8) & $8277(23.3)$ & $751(9.1)$ & & $4889(21.9)$ & $486(9.9)$ \\
\hline High (9-14) & $18091(51.0)$ & $6310(34.9)$ & & $11664(52.2)$ & $3666(31.4)$ \\
\hline Very high (215) & $6521(18.4)$ & $4320(66.2)$ & & $4158(18.6)$ & $2557(61.5)$ \\
\hline Overall & 35463 & 11426 & 22361 & 6729 \\
\hline
\end{tabular}

in patients with covid-19, with increasing number of comorbidities associated with poorer outcomes. ${ }^{16}$ In our cohort, the inclusion of individual comorbidities within the machine learning model conferred minimal additional discriminatory performance, supporting the inclusion of an overall comorbidity count.

\section{Strengths and limitations of this study}

The ISARIC WHO CCP-UK study represents a large prospectively collected cohort admitted to hospital with covid-19 and reflects the clinical data available in most economically developed healthcare settings. We derived a clinically applicable prediction score with clear methods and tested it against existing risk stratification scores in a large patient cohort admitted to hospital. The score compared favourably with other prognostic tools, with good to excellent discrimination, calibration, and performance characteristics.

The 4C Mortality Score has several methodological advantages over current covid-19 prognostic scores. The use of penalised regression methods and an eventto-variable ratio greater than 100 reduce the risk of overfitting. ${ }^{45} 46$ The use of parameters commonly available at first assessment increases its clinical applicability, avoiding the requirement for markers often only available after a patient has been admitted to a critical care facility. ${ }^{47}$ Of course a model developed in a specific dataset should describe that dataset best.

\begin{tabular}{|c|c|c|}
\hline \multirow[b]{2}{*}{ Model } & \multicolumn{2}{|c|}{ Validation cohort* } \\
\hline & No of patients with required parameters & AUROC $(95 \% \mathrm{Cl})$ \\
\hline SOFA & 197 & $0.614(0.530$ to 0.698$)$ \\
\hline qSOFA & 19361 & $0.622(0.615$ to 0.630$)$ \\
\hline Surgispheret & 18986 & $0.630(0.622$ to 0.639$)$ \\
\hline SMARTCOP & 486 & $0.645(0.593$ to 0.697$)$ \\
\hline NEWS & 19074 & $0.654(0.645$ to 0.662$)$ \\
\hline DL scoret & 16345 & 0.669 (0.660 to 0.678$)$ \\
\hline SCAP & 370 & $0.675(0.620$ to 0.729$)$ \\
\hline CRB65 & 19361 & 0.683 (0.676 to 0.691$)$ \\
\hline COVID-GRAM† & 1239 & $0.706(0.675$ to 0.736$)$ \\
\hline DS-CRB65 & 18718 & $0.718(0.710$ to 0.725$)$ \\
\hline CURB65 & 15560 & $0.720(0.713$ to 0.728$)$ \\
\hline Xie scoret & 1753 & $0.727(0.701$ to 0.753$)$ \\
\hline A-DROP & 15572 & $0.736(0.728$ to 0.744$)$ \\
\hline PSI & 360 & $0.736(0.683$ to 0.790$)$ \\
\hline E-CURB65 & 1553 & 0.764 (0.740 to 0.788$)$ \\
\hline 4C Mortality Score & 14398 & 0.774 (0.767 to 0.782$)$ \\
\hline
\end{tabular}

However, by including comparisons with pre-existing models, reassurance is provided that equivalent performance cannot be delivered with a simple tool already in use.

Additionally, in a pandemic, baseline infection rates and patient characteristics might change by time and geography. This motivated the temporal and geographical validation, which is crucial to the reporting of this study. These sensitivity analyses showed that score performance continued to be robust over time and geography.

Our study has limitations. Firstly, we were unable to evaluate the predictive performance of several existing scores that require a large number of parameters (for example, APACHE $\mathrm{II}^{48}$ ), as well as several other covid-19 prognostic scores that use computed tomography findings or uncommonly measured biomarkers. ${ }^{5}$ Additionally, several potentially relevant comorbidities, such as hypertension, previous myocardial infarction, and stroke, ${ }^{16}$ were not included in data collection. The inclusion of these comorbidities might have impacted upon or improved the performance and generalisability of the 4C Mortality Score.

Secondly, a proportion of recruited patients (3.3\%) had incomplete episodes. Selection bias is possible if patients with incomplete episodes, such as those with prolonged hospital admission, had a differential mortality risk to those with completed episodes. Nevertheless, the size of our patient cohort compares favourably to other datasets for model creation. The patient cohort on which the 4C Mortality Score was derived comprised patients admitted to hospital who were seriously ill (mortality rate of 32.2\%) and were of advanced age (median age 73 years). This model is not for use in the community and could perform differently in populations at lower risk of death. Further external validation is required to determine whether the $4 \mathrm{C}$ Mortality Score is generalisable among younger patients and in settings outside the UK.

\section{Conclusions and policy implications}

We have derived and validated an easy-to-use eight variable score that enables accurate stratification of patients with covid-19 admitted to hospital by mortality risk at hospital presentation. Application within the validation cohorts showed this tool could guide clinician decisions, including treatment escalation.

A key aim of risk stratification is to support clinical management decisions. Four risk classes were identified and showed similar adverse outcome rates across the validation cohort. Patients with a 4C Mortality Score falling within the low risk groups (mortality rate 1\%) might be suitable for management in the community, while those within the intermediate risk group were at lower risk of mortality (mortality rate $10 \% ; 22 \%$ of the cohort) and might be suitable for ward level monitoring. Similar mortality rates have been identified as an appropriate cut-off value in pneumonia risk stratification scores (CURB-65 and 

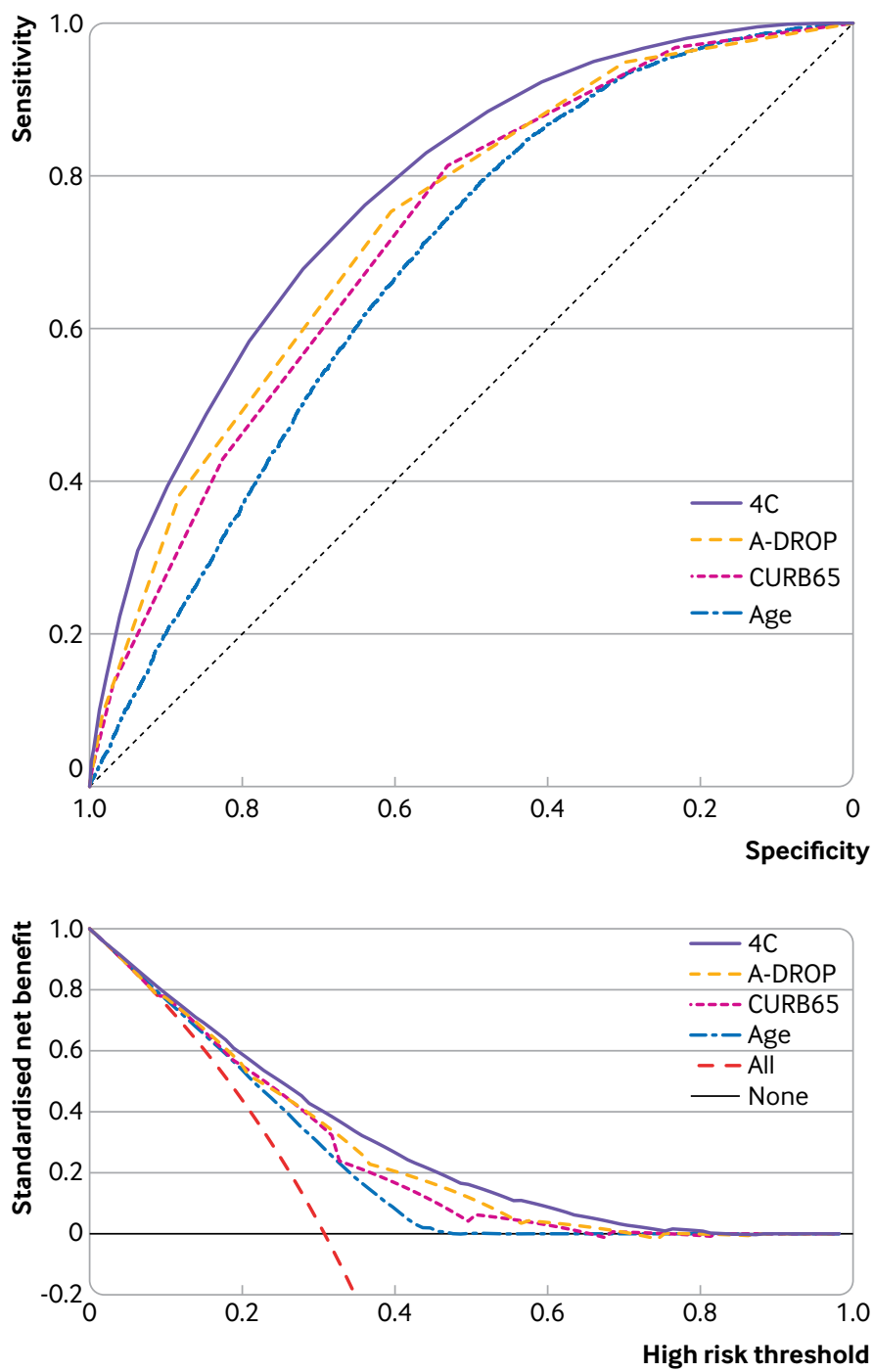

Fig 3 | Receiver operator characteristic curves (upper panel) and decision curve analysis (lower panel) for most discriminating three models applicable to more than $50 \%$ of validation population compared with age alone (restricted cubic spline; imputed datasets). In lower panel, lines are shown for standardised net benefit at different risk thresholds of treating no patients (black line) and treating all patients (red dashed line)

PSI). ${ }^{2122}$ Meanwhile patients with a score of 9 or higher were at high risk of death (around 40\%), which could prompt aggressive treatment, including the initiation of steroids ${ }^{49}$ and early escalation to critical care if appropriate.

\section{AUTHOR AFFILIATIONS}

${ }^{1}$ Centre for Medical Informatics, The Usher Institute, University of Edinburgh, Edinburgh, UK

${ }^{2}$ Medical Research Council, University of Glasgow Centre for Virus Research, Glasgow, UK

${ }^{3}$ Department of Infectious Diseases, Queen Elizabeth University Hospital, Glasgow, UK

${ }^{4}$ Institute of Population Health Sciences, University of Liverpool, Liverpool, UK

${ }^{5}$ ISARIC Global Support Centre, Centre for Tropical Medicine and Global Health, Nuffield Department of Medicine, University of Oxford, Oxford, UK

${ }^{6}$ National Infection Service, Public Health England, London, UK ${ }^{7}$ National Heart and Lung Institute, Imperial College London, London, UK
${ }^{8}$ Liverpool Clinical Trials Centre, University of Liverpool, Liverpool, UK

${ }^{9}$ Institute of Microbiology \& Infection, University of Birmingham, Birmingham, UK

${ }^{10}$ Institute of Global Health, University College London, London, UK

${ }^{11}$ NIHR Health Protection Research Unit, Institute of Infection, Veterinary and Ecological Sciences, Faculty of Health and Life Sciences, University of Liverpool, Liverpool, UK

${ }^{12}$ Division of Epidemiology and Public Health, University of Nottingham School of Medicine, Nottingham, UK

${ }^{13}$ Division of Infection and Immunity, University College London, London, UK

${ }^{14} \mathrm{Centre}$ for Tropical Medicine and Global Health, Nuffield Department of Medicine, University of Oxford, Oxford, UK

${ }^{15}$ Queen's Medical Research Institute, University of Edinburgh, Edinburgh, UK

${ }^{16}$ Walton Centre NHS Foundation Trust, Liverpool, UK

${ }^{17}$ Health Data Research UK, London, UK

${ }^{18}$ Department of Child Life and Health, University of Edinburgh, Edinburgh, UK

${ }^{19}$ Tropical \& Infectious Disease Unit, Royal Liverpool University Hospital, Liverpool, UK

${ }^{20}$ Roslin Institute, University of Edinburgh, Edinburgh, UK

${ }^{21}$ Intensive Care Unit, Royal Infirmary Edinburgh, Edinburgh, UK

${ }^{22}$ Respiratory Medicine, Alder Hey Children's Hospital, Institute in The Park, University of Liverpool, Alder Hey Children's Hospital, Liverpool L12 2AP, UK

${ }^{23}$ Department of Clinical Surgery, University of Edinburgh, Edinburgh, UK

The study protocol is available at https://isaric4c.net/protocols; study registry https://www.isrctn.com/ISRCTN66726260. This work uses data provided by patients and collected by the NHS as part of their care and support \#DataSavesLives. We are grateful to the 2648 frontline NHS clinical and research staff and volunteer medical students who collected the data in challenging circumstances; and the generosity of the participants and their families for their individual contributions in these difficult times. We also acknowledge the support of Jeremy J Farrar, Nahoko Shindo, Devika Dixit, Nipunie Rajapakse, Lyndsey Castle, Martha Buckley, Debbie Malden, Katherine Newell, Kwame O’Neill, Emmanuelle Denis, Claire Petersen, Scott Mullaney, Sue MacFarlane, Nicole Maziere, Julien Martinez, Oslem Dincarslan, and Annette Lake.

ISARIC Coronavirus Clinical Characterisation Consortium (ISARIC-4C) Investigators: consortium lead investigator: J Kenneth Baillie; chief investigator: Malcolm G Semple; co-lead investigator: Peter JM Openshaw; ISARIC clinical coordinator: Gail Carson; COinvestigators: Beatrice Alex, Benjamin Bach, Wendy S Barclay, Debby Bogaert, Meera Chand, Graham S Cooke, Annemarie B Docherty, Jake Dunning, Ana da Silva Filipe, Tom Fletcher, Christopher A Green, Ewen M Harrison, Julian A Hiscox, Antonia Ying Wai Ho, Peter W Horby, Samreen ljaz, Saye Khoo, Paul Klenerman, Andrew Law, Wei Shen Lim, Alexander J Mentzer, Laura Merson, Alison M Meynert, Mahdad Noursadeghi, Shona C Moore, Massimo Palmarini, William A Paxton, Georgios Pollakis, Nicholas Price, Andrew Rambaut, David L Robertson, Clark D Russell, Vanessa Sancho-Shimizu, Janet T Scott, Louise Sigfrid, Tom Solomon, Shiranee Sriskandan, David Stuart, Charlotte Summers, Richard S Tedder, Emma C Thomson, Ryan S Thwaites, Lance CW Turtle, Maria Zambon; project managers: Hayley Hardwick, Chloe Donohue, Jane Ewins, Wilna Oosthuyzen, Fiona Griffiths; data analysts: Lisa Norman, Riinu Pius, Tom M Drake, Cameron J Fairfield, Stephen Knight, Kenneth A Mclean, Derek Murphy, Catherine A Shaw; data and information system manager: Io Dalton, Michelle Girvan, Egle Saviciute, Stephanie Roberts, Janet Harrison, Laura Marsh, Marie Connor, Sophie Halpin, Clare Jackson, Carrol Gamble; data integration and presentation: Gary Leeming, Andrew Law, Ross Hendry, James Scott-Brown; material management: William Greenhalf, Victoria Shaw, Sarah McDonald; outbreak laboratory volunteers: Katie A Ahmed, Jane A Armstrong, Milton Ashworth, Innocent G Asiimwe, Siddharth Bakshi, Samantha L Barlow, Laura Booth, Benjamin Brennan, Katie Bullock, Benjamin WA Catterall, Jordan J Clark, Emily A Clarke, Sarah Cole, Louise Cooper, Helen Cox, Christopher Davis, Oslem Dincarslan, Chris Dunn, Philip Dyer, Angela Elliott, Anthony Evans, Lewis WS Fisher, Terry Foster, Isabel GarciaDorival, William Greenhalf, Philip Gunning, Catherine Hartley, Antonia Ho, Rebecca L Jensen, Christopher B Jones, Trevor R Jones, Shadia Khandaker, Katharine King, Robyn T Kiy, Chrysa Koukorava, Annette 
Lake, Suzannah Lant, Diane Latawiec, L Lavelle-Langham, Daniella Lefteri, Lauren Lett, Lucia A Livoti, Maria Mancini, Sarah McDonald, Laurence McEvoy, John McLauchlan, Soeren Metelmann, Nahida S Miah, Joanna Middleton, Joyce Mitchell, Shona C Moore, Ellen G Murphy, Rebekah Penrice-Randal, Jack Pilgrim, Tessa Prince, Will Reynolds, P Matthew Ridley, Debby Sales, Victoria E Shaw, Rebecca K Shears, Benjamin Small, Krishanthi S Subramaniam, Agnieska Szemiel, Aislynn Taggart, Jolanta Tanianis-Hughes, Jordan Thomas, Erwan Trochu, Libby van Tonder, Eve Wilcock, J Eunice Zhang; local principal investigators: Kayode Adeniji, Daniel Agranoff, Ken Agwuh, Dhiraj Ail, Ana Alegria, Brian Angus, Abdul Ashish, Dougal Atkinson, Shahedal Bari, Gavin Barlow, Stella Barnass, Nicholas Barrett, Christopher Bassford, David Baxter, Michael Beadsworth, Jolanta Bernatoniene, John Berridge, Nicola Best, Pieter Bothma, David Brealey, Robin Brittain-Long, Naomi Bulteel, Tom Burden, Andrew Burtenshaw, Vikki Caruth, David Chadwick, Duncan Chambler, Nige Chee, Jenny Child, Srikanth Chukkambotla, Tom Clark, Paul Collini, Catherine Cosgrove, Jason Cupitt, Maria-Teresa Cutino-Moguel, Paul Dark, Chris Dawson, Samir Dervisevic, Phil Donnison, Sam Douthwaite, Ingrid DuRand, Ahilanadan Dushianthan, Tristan Dyer, Cariad Evans, Chi Eziefula, Chrisopher Fegan, Adam Finn, Duncan Fullerton, Sanjeev Garg, Sanjeev Garg, Atul Garg, Jo Godden, Arthur Goldsmith, Clive Graham, Elaine Hardy, Stuart Hartshorn, Daniel Harvey, Peter Havalda, Daniel B Hawcutt, Maria Hobrok, Luke Hodgson, Anita Holme, Anil Hormis, Michael Jacobs, Susan Jain, Paul Jennings, Agilan Kaliappan, Vidya Kasipandian, Stephen Kegg, Michael Kelsey, Jason Kendall, Caroline Kerrison, Ian Kerslake, Oliver Koch, Gouri Koduri, George Koshy, Shondipon Laha, Susan Larkin, Tamas Leiner, Patrick Lillie, lames Limb, Vanessa Linnett, Jeff Little, Michael MacMahon, Emily MacNaughton, Ravish Mankregod, Huw Masson, Elijah Matovu, Katherine McCullough, Ruth McEwen, Manjula Meda, Gary Mills, Jane Minton, Mariyam Mirfenderesky, Kavya Mohandas, Quen Mok, James Moon, Elinoor Moore, Patrick Morgan, Craig Morris, Katherine Mortimore, Samuel Moses, Mbiye Mpenge, Rohinton Mulla, Michae Murphy, Megan Nagel, Thapas Nagarajan, Mark Nelson, Igor Otahal, Mark Pais, Selva Panchatsharam, Hassan Paraiso, Brij Patel, Justin Pepperell, Mark Peters, Mandeep Phull, Stefania Pintus, Jagtur Singh Pooni, Frank Post, David Price, Rachel Prout, Nikolas Rae, Henrik Reschreiter, Tim Reynolds, Neil Richardson, Mark Roberts, Devender Roberts, Alistair Rose, Guy Rousseau, Brendan Ryan, Taranprit Saluja, Aarti Shah, Prad Shanmuga, Anil Sharma, Anna Shawcross, Jeremy Sizer, Richard Smith, Catherine Snelson, Nick Spittle, Nikki Staines, Tom Stambach, Richard Stewart, Pradeep Subudhi, Tamas Szakmany, Kate Tatham, Jo Thomas, Chris Thompson, Robert Thompson, Ascanio Tridente, Darell Tupper-Carey, Mary Twagira, Andrew Ustianowski, Nick Vallotton, Lisa Vincent-Smith, Shico Visuvanathan, Alan Vuylsteke, Sam Waddy, Rachel Wake, Andrew Walden, Ingeborg Welters, Tony Whitehouse, Paul Whittaker, Ashley Whittington, Meme Wijesinghe, Martin Williams, Lawrence Wilson, Sarah Wilson, Stephen Winchester, Martin Wiselka, Adam Wolverson, Daniel G Wooton, Andrew Workman, Bryan Yates, Peter Young.

Contributors: Conceptualisation: SRK, AH, RP, GC, JD, PWH, LM, JSN-V-T, CS, PJMO, JKB, MGS, ABD, EMH; data curation: RP, SH, KAH, C), CAG, KAM, LM, LN, CDR, CAS, MGS, ABD, EMH; formal analysis: SRK, AH, RP, TMD, CJF, KAM, MGP, LCWT, EMH. Funding acquisition: PWH, JSN-V-T, TS, PJMO, JKB, MGS, ABD. Investigation: SRK, AH, CDR, OVS, LCWT, PJMO, JKB, MGS, ABD, EMH; methodology: SRK, AH, RP, IB, KAM, CAS, AS, CS, ABD, EMH; project administration: $\mathrm{SH}, \mathrm{HEH}, \mathrm{CJ}, \mathrm{LM}, \mathrm{LN}$, CDR, CAS, MGS; resources: CG, PWH, LM, TS, MGS, EMH; software: SRK, RP, KAM, OVS; supervision: SRK, RP, CG, HEH, PWH, PLO, PJMO, JKB, MGS, EMH; visualisation: SRK, RP, MGP, EMH; writing-original draft: SRK, EMH; writing-review and editing: SRK, RG, AH, RP, IB, GC, TMD, CAG, JD, CJF, LM, MN, JSN-V-T, PLO, MGP, CDR, AS, CS, OVS, LCWT, PJMO, JKB, MGS, ABD, EMH. SRK and AH are joint first authors. $M G S, A B D$, and $E M H$ are joint senior authors. MGS is guarantor and corresponding author for this work, and attests that all listed authors meet authorship criteria and that no others meeting the criteria have been omitted.

Funding: This work is supported by grants from: the National Institute for Health Research (NIHR; award CO-CIN-01), the Medical Research Council (MRC; grant MC_PC_19059), and by the NIHR Health Protection Research Unit (HPRU) in Emerging and Zoonotic Infections at University of Liverpool in partnership with Public Health England (PHE), in collaboration with Liverpool School of Tropical Medicine and the University of Oxford (award 200907), NIHR HPRU in Respiratory Infections at Imperial College London with PHE (award 200927), Wellcome Trust and Department for International Development (DID; 215091/Z/18/Z), the Bill and Melinda Gates Foundation (OPP1209135), Liverpool Experimental Cancer Medicine Centre (grant reference C18616/A25153), NIHR Biomedical Research Centre at Imperial College London (IS-BRC-1215-20013), EU Platform for European Preparedness Against (Re-)emerging Epidemics (PREPARE; FP7 project 602525), and NIHR Clinical Research Network for providing infrastructure support for this research. PJMO is supported by a NIHR senior investigator award (201385). LT is supported by the Wellcome Trust (award 205228/Z/16/Z). MN is funded by a WT investigator award (207511/Z/17/Z) and the NIHR University College London Hospitals Biomedical Research Centre. The views expressed are those of the authors and not necessarily those of the Department of Health and Social Care, DID, NIHR, MRC, Wellcome Trust, or PHE. Competing interests: All authors have completed the ICMJE uniform disclosure form at www.icmje.org/coi_disclosure.pdf and declare: support from the National Institute for Health Research (NIHR), the Medical Research Council (MRC), the NIHR Health Protection Research Unit (HPRU) in Emerging and Zoonotic Infections at University of Liverpool, Public Health England (PHE), Liverpool School of Tropical Medicine, University of Oxford, NIHR HPRU in Respiratory Infections at Imperial College London, Wellcome Trust, Department for International Development, the Bill and Melinda Gates Foundation, Liverpool Experimental Cancer Medicine Centre, NIHR Biomedical Research Centre at Imperial College London, EU Platform for European Preparedness Against (Re-)emerging Epidemics (PREPARE), and NIHR Clinical Research Network for the submitted work; ABD reports grants from Department of Health and Social Care (DHSC), during the conduct of the study, grants from Wellcome Trust, outside the submitted work; CAG reports grants from DHSC National Institute of Health Research (NIHR) UK, during the conduct of the study; PWH reports grants from Wellcome Trust, Department for International Development, Bill and Melinda Gates Foundation, NIHR, during the conduct of the study; JSN-V-T reports grants from DHSC, England, during the conduct of the study, and is seconded to DHSC, England; MN is supported by a Wellcome Trust investigator award and the NIHR University College London Hospitals Biomedical Research Centre (BRC); PJMO reports personal fees from consultancies and from European Respiratory Society, grants from MRC, MRC Global Challenge Research Fund, EU, NIHR BRC, MRC/GSK, Wellcome Trust, NIHR (Health Protection Research Unit (HPRU) in Respiratory Infection), and is NIHR senior investigator outside the submitted work; his role as President of the British Society for Immunology was unpaid but travel and accommodation at some meetings was provided by the Society; JKB reports grants from MRC UK; MGS reports grants from DHSC NIHR UK, grants from MRC UK, grants from HPRU in Emerging and Zoonotic Infections, University of Liverpool, during the conduct of the study, other from Integrum Scientific LLC, Greensboro, NC, USA, outside the submitted work; LCWT reports grants from HPRU in Emerging and Zoonotic Infections, University of Liverpool, during the conduct of the study, and grants from Wellcome Trust outside the submitted work; EMH, HEH, JD, RG, RP, LN, KAH, GC, LM, SH, C), and CG, all declare: no support from any organisation for the submitted work; no financial relationships with any organisations that might have an interest in the submitted work in the previous three years; and no other relationships or activities that could appear to have influenced the submitted work. Ethical approval: Ethical approval was given by the South CentralOxford C Research Ethics Committee in England (reference 13/ SC/0149), and by the Scotland A Research Ethics Committee (reference 20/SS/0028). The study was registered at https://www. isrctn.com/ISRCTN66726260.

Data sharing: We welcome applications for data and material access via our Independent Data and Material Access Committee (https:// isaric4c.net).

The lead author (the manuscript's guarantor) affirms that the manuscript is an honest, accurate, and transparent account of the study being reported; that no important aspects of the study have been omitted; and that any discrepancies from the study as planned (and, if relevant, registered) have been explained.

Dissemination to participants and related patient and public communities: ISARIC-4C has a public facing website isaric 4 c.net and twitter account (@CCPUKstudy).We are engaging with print and internet press, television, radio, news, and documentary programme makers. We will explore distribution of findings with The Asthma UK and British Lung Foundation Partnership and take advice from NIHR Involve and GenerationR Alliance Young People's Advisory Groups.

Provenance and peer review statement: Not commissioned; externally peer reviewed.

This is an Open Access article distributed in accordance with the terms of the Creative Commons Attribution (CC BY 4.0) license, which 
permits others to distribute, remix, adapt and build upon this work, for commercial use, provided the original work is properly cited. See: http://creativecommons.org/licenses/by/4.0/.

1 Yang X, Yu Y, Xu J, et al. Clinical course and outcomes of critically ill patients with SARS-CoV-2 pneumonia in Wuhan, China: a single-centered, retrospective, observational study. Lancet Respir Med 2020;8:475-81. doi:10.1016/S2213-2600(20)30079-5

2 Johns Hopkins Coronavirus Resource Center. COVID-19 map. 2020. https://coronavirus.jhu.edu/map.html

3 WHO. COVID-19 situation reports. 2020. https://www.who.int/ emergencies/diseases/novel-coronavirus-2019/situation-reports

4 Tolksdorf K, Buda S, Schuler E, Wieler LH, Haas W. Influenzaassociated pneumonia as reference to assess seriousness of coronavirus disease (COVID-19). Euro Surveill 2020;25:2000258. doi:10.2807/1560-7917.ES.2020.25.11.2000258

5 Wynants L, Calster BV, Bonten MMJ, et al. Prediction models for diagnosis and prognosis of covid-19 infection: systematic review and critical appraisal. 2020. https://www.bmj.com/content/369/bmj. m1328

6 Chen J-H, Chang S-S, Liu JJ, et al. Comparison of clinical characteristics and performance of pneumonia severity score and CURB-65 among younger adults, elderly and very old subjects. Thorax 2010;65:9717. doi:10.1136/thx.2009.129627

7 Gupta RK, Marks M, Samuels THA, Luintel A, Rampling T, Chowdhury $\mathrm{H}$, et al. Systematic evaluation and external validation of 22 prognostic models among hospitalised adults with COVID-19: an observational cohort study. medRxiv. 2020. doi:10.1101/2020.07.2 4.20149815

8 Docherty AB, Harrison EM, Green CA, et al, ISARIC4C Investigators. Features of 20133 UK patients in hospital with covid-19 using the ISARIC WHO Clinical Characterisation Protocol: prospective observational cohort study. 2020. https://www.bmj.com/ content/369/bmj.m1985

9 Collins GS, Reitsma JB, Altman DG, Moons KGM. Transparent reporting of a multivariable prediction model for individual prognosis or diagnosis (TRIPOD): the TRIPOD statement. BMJ 2015;350:g7594. doi:10.1136/bmj.g7594

10 Charlson ME, Pompei P, Ales KL, MacKenzie CR. A new method of classifying prognostic comorbidity in longitudinal studies: development and validation. J Chronic Dis 1987;40:373-83. doi:10.1016/0021-9681(87)90171-8

11 Sattar N, McInnes IB, McMurray JJV. Obesity Is a Risk Factor for Severe COVID-19 Infection: Multiple Potential Mechanisms. Circulation 2020;142:4-6. doi:10.1161/ CIRCULATIONAHA.120.047659

12 Simonnet A, Chetboun M, Poissy J, et al, LICORN and the Lille COVID-19 and Obesity study group. High prevalence of obesity in severe acute respiratory syndrome coronavirus-2 (SARSCoV-2) requiring invasive mechanical ventilation. Obesity (Silver Spring) 2020;28:1195-9. doi:10.1002/oby.22831

13 Dunning JW, Merson L, Rohde GGU, et al, ISARIC Working Group 3, ISARIC Council. Open source clinical science for emerging infections. Lancet Infect Dis 2014;14:8-9. doi:10.1016/S1473 3099(13)70327-X

14 Riley RD, Ensor J, Snell KIE, et al. Calculating the sample size required for developing a clinical prediction model. 2020. https://www.bmj. com/content/368/bmj.m441

15 Moons KGM, Altman DG, Reitsma JB, et al. Transparent Reporting of a multivariable prediction model for Individual Prognosis or Diagnosis (TRIPOD): explanation and elaboration. Ann Intern Med 2015;162:W1-73. doi:10.7326/M14-0698

16 Guan W, Liang W, Zhao Y, et al. Comorbidity and its impact on 1590 patients with COVID-19 in China: a nationwide analysis. European Respiratory Journal. 2020. https://erj.ersjournals.com/ content/55/5/2000547

17 Rubin DB, editor. Multiple imputation for nonresponse in surveys. 1987. http://doi.wiley.com/10.1002/9780470316696

18 Barrio I, Arostegui I, Quintana JM, Group IC, Group I-C. Use of generalised additive models to categorise continuous variables in clinical prediction. BMC Med Res Methodol 2013;13:83. doi:10.1186/1471-2288-13-83

19 Mandrekar JN. Receiver operating characteristic curve in diagnostic test assessment. / Thorac Oncol 2010;5:1315-6. doi:10.1097/ JTO.0b013e3181ec173d

20 Steyerberg EW, Vickers AJ, Cook NR, et al. Assessing the performance of prediction models: a framework for traditional and novel measures. Epidemiology 2010;21:128-38. doi:10.1097/ EDE.0b013e3181c30fb2

21 Lim WS, van der Eerden MM, Laing R, et al. Defining community acquired pneumonia severity on presentation to hospital: an international derivation and validation study. Thorax 2003;58:37782. doi:10.1136/thorax.58.5.377
22 Fine MJ, Auble TE, Yealy DM, et al. A prediction rule to identify low-risk patients with community-acquired pneumonia. N Engl/ Med 1997:336:243-50 doi:10.1056/NEIM199701233360402

23 Hacking JM, Muller S, Buchan IE. Trends in mortality from 1965 to 2008 across the English north-south divide: comparative observational study. 2011. https://www.ncbi.nlm.nih.gov/pmc/ articles/PMC3039695/

24 Buchan IE, Kontopantelis E, Sperrin M, Chandola T, Doran T. NorthSouth disparities in English mortality1965-2015: longitudinal population study. J Epidemiol Community Health 2017;71:928-36. doi:10.1136/jech-2017-209195

25 Office for National Statistics. Deaths involving COVID-19 by local area and socioeconomic deprivation. 2020 https://www.ons.gov.uk/ peoplepopulationandcommunity/birthsdeathsandmarriages/deaths/ bulletins/deathsinvolvingcovid19bylocalareasanddeprivation/ deathsoccurringbetween 1 marchand17april

26 Harrison EM, Docherty AB, Barr B, et al, ISARIC4C Investigators. Ethnicity and Outcomes from COVID-19. The ISARIC CCP-UK Prospective Observational Cohort Study of Hospitalised Patients. 2020. https://papers.ssrn.com/sol3/papers.cfm?abstract_ id $=3618215$

27 Vickers AJ, Elkin EB. Decision curve analysis: a novel method for evaluating prediction models. Med Decis Making 2006;26:565-74. doi:10.1177/0272989X06295361

28 Miyashita N, Matsushima T, Oka M, Japanese Respiratory Society. The JRS guidelines for the management of community-acquired pneumonia in adults: an update and new recommendations. Intern Med 2006;45:419-28. doi:10.2169/internalmedicine.45.1691

29 Bauer TT, Ewig S, Marre R, Suttorp N, Welte T, CAPNETZ Study Group. CRB-65 predicts death from community-acquired pneumonia. J Intern Med 2006;260:93-101. doi:10.1111/j.1365-2796.2006.01657.x

30 Dwyer R, Hedlund J, Henriques-Normark B, Kalin M. Improvement of CRB-65 as a prognostic tool in adult patients with communityacquired pneumonia. BMJ Open Respir Res 2014;1:e000038. doi:10.1136/bmjresp-2014-000038

31 Liu IL, Xu F, Zhou H, et al. Expanded CURB-65: a new score system predicts severity of community-acquired pneumonia with superior efficiency. Sci Rep 2016;6:22911. doi:10.1038/srep22911

32 RCP London. National Early Warning Score (NEWS) 2. 2017. https:// www.rcplondon.ac.uk/projects/outputs/national-early-warningscore-news-2

33 Singer M, Deutschman CS, Seymour CW, et al. The Third International Consensus Definitions for Sepsis and Septic Shock (Sepsis-3). JAMA 2016;315:801-10. doi:10.1001/jama.2016.0287

34 Charles PGP, Wolfe R, Whitby M, et al, Australian CommunityAcquired Pneumonia Study Collaboration. SMART-COP: a tool for predicting the need for intensive respiratory or vasopressor support in community-acquired pneumonia. Clin Infect Dis 2008;47:375-84 doi:10.1086/589754

35 Vincent JL, Moreno R, Takala J, et al. The SOFA (Sepsis-related Organ Failure Assessment) score to describe organ dysfunction/failure. On behalf of the Working Group on Sepsis-Related Problems of the European Society of Intensive Care Medicine. Intensive Care Med 1996;22:707-10. doi:10.1007/BF01709751

36 Yandiola PPE, Capelastegui A, Quintana J, et al. Prospective comparison of severity scores for predicting clinically relevant outcomes for patients hospitalized with communityacquired pneumonia. Chest 2009;135:1572-9. doi:10.1378/ chest.08-2179

37 Liang W, Liang H, Ou L, et al. Development and validation of a clinical risk score to predict the occurrence of critical illness in hospitalized patients with COVID-19. 2020. https://jamanetwork.com/journals/ jamainternalmedicine/fullarticle/2766086

38 Xie J, Hungerford D, Chen H, Abrams ST, Li S, Wang G, et al. Development and external validation of a prognostic multivariable model on admission for hospitalized patients with COVID-19. medRxiv.2020. doi:10.1101/2020.03.28.20045997

39 Businesswire. Surgisphere's COVID-19 machine learning platform receives international endorsement - now clinically in use at >1,000 hospitals worldwide. 2020. https://www.businesswire.com/news/ home/20200326005199/en/Surgisphere\%E2\%80\%99s-COVID19-Machine-Learning-Platform-Receives-International

40 Zhang H, Shi T, Wu X, Zhang Z, Wang K, Bean D, et al. Risk prediction for poor outcome and death in hospital in-patients with COVID-19: derivation in Wuhan, China and external validation in London, UK. medRxiv. 2020. doi:10.1101/2020.04.28.20082222

41 Caramelo F, Ferreira N, Oliveiros B. Estimation of risk factors for COVID-19 mortality - preliminary results. medRxiv. 2020. doi:10.110 1/2020.02.24.20027268

42 Williams RD, Markus AF, Yang C, Salles TD, Falconer T, Jonnagaddala J, et al. Seek COVER: Development and validation of a personalized risk calculator for COVID-19 outcomes in an international network. medRxiv. 2020. doi:10.1101/2020.05.26.20112649 
43 Luo X, Zhou W, Yan X, et al. Prognostic value of C-reactive protein in patients with COVID-19. 2020. https://academic.oup.com/cid/ advance-article/doi/10.1093/cid/ciaa641/5843450

44 Sterne JAC, White IR, Carlin JB, et al. Multiple imputation for missing data in epidemiological and clinical research: potential and pitfalls. 2009. https://www.bmi.com/content/338/bmj.b2393

45 Pavlou M, Ambler G, Seaman SR, et al. How to develop a more accurate risk prediction model when there are few events. 2015 https://www.ncbi.nlm.nih.gov/pmc/articles/PMC4531311/

46 Peduzzi P, Concato J, Feinstein AR, Holford TR. Importance of events per independent variable in proportional hazards regression analysis. II. Accuracy and precision of regression estimates. J Clin Epidemiol 1995;48:1503-10. doi:10.1016/0895 4356(95)00048-8

47 Song Y, Zheng S, Li L, Zhang X, Zhang X, Huang Z, et al. Deep learning enables accurate diagnosis of novel coronavirus (COVID-19) with CT images. medRxiv. 2020. doi:10.1101/2020.02.23.20026930

48 Knaus WA, Draper EA, Wagner DP, Zimmerman JE. APACHE II: a severity of disease classification system. Crit Care Med 1985;13:81829. doi:10.1097/00003246-198510000-00009

49 Welcome - RECOVERY Trial. 2020. https://www.recoverytrial.net/

Web appendix: Supplementary material 